\title{
Storage Bins: Mobile Storage for Collaborative Tabletop Displays
} \author{
Interactions Laboratory
Department of Computer Science \\ University of Calgary \\ 2500 University Drive NW \\ Calgary, AB T2N 1N4 \\ Canada \\ $\{$ sdscott, sheelagh\}@cpsc.ucalgary.ca
}

Stacey D. Scott, M. Sheelagh T. Carpendale, Stefan Habelski,

\begin{abstract}
Casual piling of paper-based media during traditional tabletop collaboration is an important practice that helps coordinate task and group interactions. Previous research has shown that the ability to move piles around on a table plays a critical role in accessing and sharing task resources. However, existing casual storage techniques for digital workspaces only provide access to stored items at the periphery of the workspace, potentially compromising collaborative interactions at a digital tabletop display. To address this issue, we introduce storage bins, a mobile storage mechanism that enables access to stored items anywhere in the workspace. We also present an exploratory user study involving the use of mobile and peripheral storage mechanisms on a large, hi-resolution collaborative tabletop display. Findings from this study demonstrate the utility of storage bins and further our understanding of the impact of mobile and peripheral storage mechanisms on collaboration at a tabletop display.
\end{abstract}

\section{Introduction}

Traditional tables have long been the preferred work environment for many collaboration tasks such as planning, scheduling, brainstorming, design and layout activities. Unfortunately, collaborating at current digital tabletop displays is often awkward and frustrating. This may, in part, stem from a lack of suitable tools for organizing and sharing information. In order to address this deficiency, we introduce a new an interface component, called storage bins, designed to support the casual storage of workspace items during collaboration on a tabletop displays.

While interest in tabletop displays began over a decade ago [11], recent technological advances have increased the feasibility of tabletop displays and fueled renewed interest in this research direction $[5,8,9]$. Borrowing ideas from tiled-projector high-resolution walls [2] and utilizing recent touch-input technology (www.smarttech.com), we have assembled a tabletop display that is large ( $4 \times 5$ feet), high- resolution (2048x1280) and supports input from two simultaneous touches. However, constructing a tabletop display is only the first step to providing interactive support for collaborative tasks. It quickly becomes apparent that accessing digital information via a standard software interface displayed on the tabletop surface can be very awkward for a single user and extremely frustrating for a group of users.

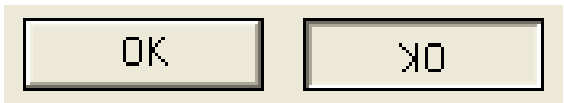

Figure 1. State ambiguity is introduced by viewing a button from opposite sides of a tabletop display.

While increasing the size of the display surface offers more space for collaborative tasks, standard software interfaces that place frequently accessed items at the display's edges can cause these items to be physically difficult to reach. Further altering the display by placing it horizontally - on a tabletop - introduces orientation issues because people can approach the display from different sides. Textual information can be difficult to read when viewed upside down or at an angle. Furthermore, the state of standard interface components can become ambiguous when viewed from different angles. For example, the same button can look ready to press when viewed right way up, yet look depressed when viewed upside-down (Figure 1). Thus, the fundamental components of tabletop interfaces need to be reconsidered.

Our approach to this issue has been to observe how people interact when using traditional, physically-based media during tabletop collaboration in order to understand what activities and interactions tabletop groupware should support and to ground our interface designs in these observations. We have studied collaborators' tabletop interactions during game playing $[3,7]$ and collaborative design [7] activities. 
During these investigations, we have repeatedly observed the practice of casual piling to facilitate organization and sharing of task resources. This practice involves creating piles, moving piles out of the way, and relocating piles for easier immediate access. Piles were often placed in the periphery of working areas when the contents of the pile were not currently in use. People also piled items elsewhere in the workspace, actively using the contents of a pile to perform their main activity. For example, during a furniture layout task, collaborators moved piles of paper furniture icons around in the main work area so that different group members could obtain items from the pile as they were creating furniture arrangements [7] (Figure 2).

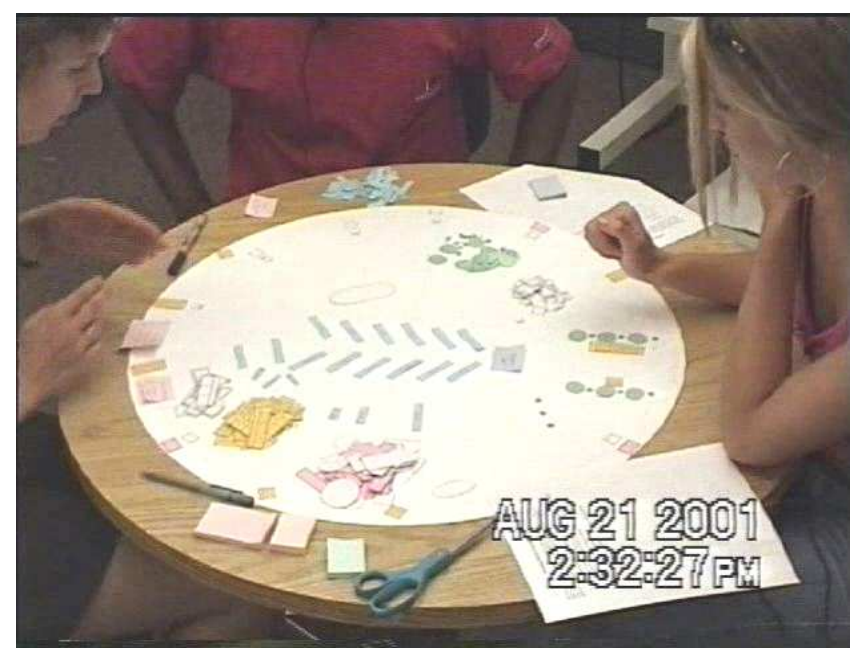

Figure 2. Piling during tabletop collaboration.

The ability to relocate piles on the table played a key role in coordinating task and group interactions during the collaborative activities. In general, when a pile of items was moved close to a person (i.e. in their personal territory [7]), the pile was reserved for use by that person. In contrast, when a pile of items was moved to a more central location, such as the middle of the table or along the edge between group members' positions at the table (i.e. in the group territory [7]), the pile was typically available for use by all group members. People also took advantage of piling items on convenient and easily movable surfaces, such as the inside of a puzzle box lid to facilitate access to resources where and when they need them, without disturbing other materials in the workspace.

The practice of piling has also been found to be useful for individual work. Piles help people organize their work, remind each other of work still to be done, and provide a cognitively light-weight mechanism for storing items that are otherwise difficult to classify [4]. During collaboration, such light-weight organizational methods allow people to focus on the main task activity and their interactions with other group members instead of having to spend time and effort on repeatedly filing and re-filing task resources.

Current techniques for casually storing items in a digital workspace, such as the ZoomScape [2] and Scalable Fabric [6], provide partial support for the practice of piling during tabletop collaboration. These techniques allow digital items to be stored in an area peripheral to the main workspace. In ZoomScape, any item on an interactive wall display can be placed into a storage area that spans the top of the display. In Scalable Fabric, desktop application windows can be placed in a storage area surrounding the main workspace to facilitate task switching. Both of these storage mechanisms shrink items as they are placed in the storage area, minimizing the space occupied by stored items. Reducing the size of stored items can facilitate searching for items in a pile because small items can easily be spread out in the available space, reducing occlusion.

However, these storage mechanisms only allow items to be stored on the periphery on the workspace. As mentioned above, the mobility of piles is critical for coordinating task and group interactions on a table. To enable this important practice in a digital tabletop workspace, we developed a mobile storage mechanism, called a storage bin, which provides the space-preserving features of existing peripheral storage mechanisms, while also providing the capability to relocate stored items in the workspace.

\section{Storage bin basics}

Combining the design criteria that arose from our initial observational studies with participant responses during pilot studies, our storage bins have the following characteristics.

Container capabilities. With physically-based media, piles were sometimes created rapidly through large hand gestures that swept items together. They were also created gradually one item at a time during different types of organizational activities. In reverse sometimes all items in a pile were effectively 'dumped' back into the workspace or alternatively one or two items were removed to fill a particular need at the time. With digital media, grouping and ungrouping actions might fill the all-in all-out piling actions but would require many selection/de-selection actions for the one or two in and out usage. Therefore storage bins provide the capability of a container, allowing items to be added or removed as a group or individually.

Mobility. Our previous observations indicate that the location of a pile is strongly connected to its relationship to the main task and that piles are often moved. Piles are often moved when someone wants to have easier access to their contents or when a pile is in the way of the main task. Extending the container metaphor, all items in a storage bin 
(a)

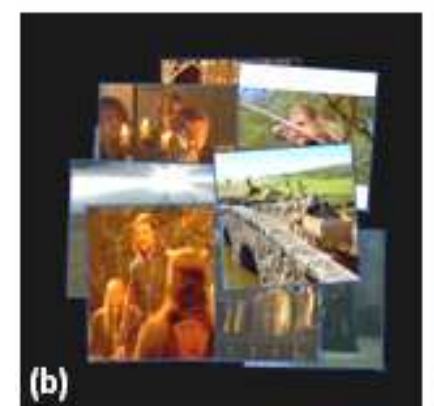

Figure 3. Storing an item in a storage bin (a). The same pile of photos are shown at full-size in (b) and stored in a storage bin (c).

could be readily relocated simply by moving the storage bin.

Visual characteristics. Storage and access from storage are peripheral activities. These activities should support the main task but not pull attention away from it. Therefore our storage bins are merely visually distinguishable from the background rather than being visually dominant. With physically-based media the shape of piles was always variant, loose, amorphous and often changing. Similarly storage bins have loose, curved and adjustable boundaries. Figure $3 \mathrm{c}$ shows a storage bin; note the casual shape and the minimal colour usage.

Storage. While in the physical world an object's size is usually constant, in the virtual world this characteristic has more flexibility. As reducing an object's size in storage has been shown to be useful $[2,6]$, the size of stored items is reduced as they enter a storage bin. Pilot tests both confirmed the usefulness of this feature and refined it by indicating that while, in general, reduction in size proportional to the objects original size (35\%) was most understandable, a minimum size (80x80 pixels) was necessary to maintain recognizability. Items are considered to have entered the storage bin when the touch point is within the storage bin. Figure 3 a illustrates the act of storing an item in a storage bin and Figures $2 \mathrm{~b}$ and $2 \mathrm{c}$ show two views of the same pile of 10 images demonstrating how the size reduction can help with occlusion: the fullsized images (b) have considerable overlap and the reduced-sized images in a storage bin (c) are not occluded.
Introducing scaling requires consideration of how and when it should occur. Pilot tests revealed that people found abrupt scaling jarring. Animated transitions can be implemented spatially, as an item crosses a region, or temporally, during a short period of time upon entering the storage bin. Adding a rim to the storage bin provides a spatial transition zone. It is possible for a part of an item to be over the storage bin without the item being considered to be inside it - an item is not stored unless the touch point enters the bin. If a subsequent touch is on a region of the item that is over the storage bin, we apply a timed scaling animation to prevent an abrupt scaling change since the touch point did not cross the transition zone.

In summary, a storage bin is a graphical user interface container component that can be used to hold other workspace items, such as images, documents, and thumbnails. Its mobility and adjustability in size and shape allows people to share resources and transition between resources at will. Moving a storage bin allows a person to bring a collection of stored items in and out of the current focus of activity. Expanding and collapsing a storage bin allow people to dynamically customize their working area: when they are actively using a collection of stored items, the storage bin can be expanded to provide easier access those items; when they are finished with the collection, the storage bin can be collapsed to free up that area of the workspace.

\section{Exploratory User Study}

In order to investigate the usability of storage bins and to understand the advantages and disadvantages of both mobile and peripheral storage mechanisms, we conducted an exploratory user study. In this study, six pairs of university students ( 3 male groups and 3 female groups) performed a collaborative photo layout task on our digital tabletop containing either storage bins or a peripheral storage area that spanned the perimeter of the tabletop workspace.

\section{Layout task}

Participants were asked to perform several photo layouts in a tabletop groupware application. During each task trial, participants were provided four theme pages $(512 \times 512$ pixels each) and 100 photos ( $125 \times 125$ or $256 \times 256$ pixels each) loosely clustered in the middle of the tabletop workspace. The photos and layout themes used for each task trial related to a popular television show or movie. The goal of each task trial was to create a photo layout for each of the four theme pages in the allotted time. Figure 4 shows an example of a layout being created on the 'Romance' theme page from the 'Friends' TV show. 


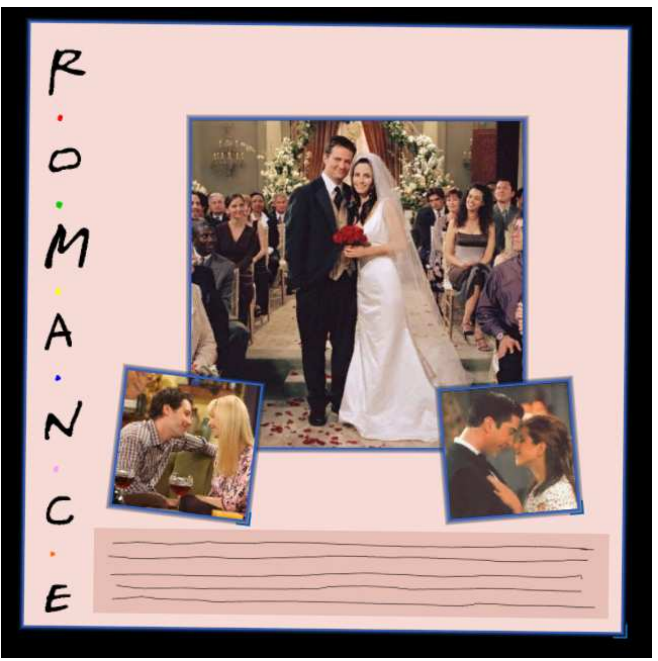

Figure 4. A photo layout being created on the theme page for 'Romance'.

\section{Collaborative tabletop workspaces}

Participants performed the layout task using two different digital tabletop workspaces: one containing several storage bins, and one containing a peripheral storage area.

Figures $2 \mathrm{c}, 5,7$, and 9 show the storage bins used in this study. Nine storage bins were provided in the workspace: one in each corner and five clustered directly between the participants' initial seating positions. The latter five storage bins were intentionally positioned between collaborators, and likely 'in the way,' because we were interested in understanding if people would move them to a more 'suitable' location and, if so, where that would be.

Figures 3 and 4 show the peripheral storage area used in this study. Storing an item (or group) in the peripheral storage area is identical to storing an item (or group) in a storage bin. Unlike the storage bins, though, the peripheral storage area is permanently fixed to the workspace edge. A larger or smaller storage area can be created by resizing the peripheral storage area. Each side can be resized

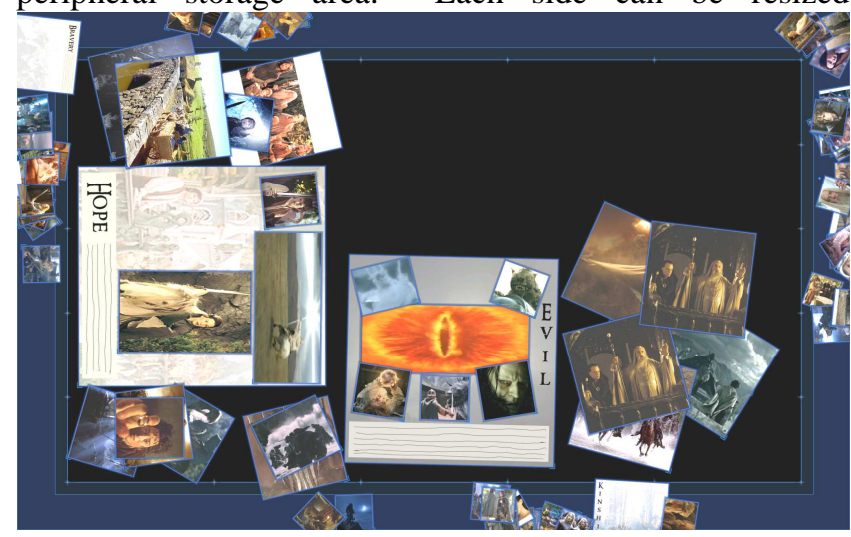

Figure 5. A screen shot of the peripheral storage area. independently to allow different sized storage areas on each side of the table.

The tabletop groupware application used for this study was implemented in Microsoft Visual C\# and OpenGL, using the Tao.OpenGL library (www.taoframework.com). The application ran on a Xeon ${ }^{\mathrm{TM}} 2.80 \mathrm{GHz}$ Windows XP PC and was displayed on our high-resolution tabletop display (described above). To provide software support for multiple users at the tabletop display Tse's DViTtoolkit (an extension of the SDGToolkit [10]) was used.

Aside from the storage mechanisms described above, the tabletop groupware contained several features useful for performing the layout task. The photos and theme pages could be easily resized via a resize handle on the lower right corner of each item. Groups could be created and selected by dragging a bounding box around several items. To address tabletop orientation issues, interactions with items or groups of items used a mechanism called Rotate 'N Translate (RNT) [3]. RNT allows an object to be simultaneously rotated and translated in a single fluid motion using a single touch point. RNT also provides the ability to toss items across the workspace with a simple 'flick' action performed on an item, allowing items to be easily passed to someone else or discarded across the table.

\section{Procedure}

Participants sat at adjacent sides of the table during the study and stood when it was necessary to reach something across the table. One pair ended up standing on opposite sides of the table for the majority of one of their sessions. All pairs performed the layout task using both storage mechanisms. Half of the groups used the peripheral storage area first, while the other half used the storage bins first. For each storage mechanism, each group was first shown how to use the storage mechanism and was then given 15 minutes to perform a practice session involving one theme page and 45 photos. In the actual task trial, the group was given 20 minutes to create the four theme layouts.

Participants' interactions in the workspace were logged to a data file and captured on videotape. Participants also completed a questionnaire after each experimental trial to elicit reactions to each storage mechanism, as well as a post-experiment questionnaire to obtain overall opinions once participants had used both storage mechanisms.

\section{Findings}

In general, participants found the storage mechanisms helpful for performing the layout activity. Both types of storage mechanisms were used frequently during the layout sessions. At least half of the 104 photos and layout pages were stored at some stage during the twelve layout sessions. In nine of the sessions at least $98 \%$ of the items 
were stored and in six sessions all 104 workspace items were stored.

While both storage mechanisms were used frequently for storing and retrieving items, the storage bins were manipulated more often than the peripheral storage area. Only one pair adjusted the peripheral storage area at all. This group enlarged the storage area in front of each person by about $25 \%$ (see Figure $6 a$ ) at the beginning of their trial and left it that way for their entire session. Conversely, the storage bins were manipulated by all six groups. The move feature was used most often: across all six trials, they were moved 75 times. How often the storage bins were moved varied widely between groups: some groups moved them only a few times, while other groups moved them over 20 times. The reshape and resize features were used much less often, but were extremely helpful when used: the storage bins were reshaped twice and resized eleven times across all trials.

Analysis of participant behaviour revealed that two distinct types of activities emerged during the layout task: coarsegrained organization and fine-grained organization. Each trial began with roughly ten minutes of coarse-grained organization of the workspace where the photos were sorted into theme piles. The second stage of the trial involved actually assembling the photo layouts. This stage involved more fine-grained organizational activities such as arranging the theme pages and candidate photos in the workspace, passing and sharing items, and manipulating the storage mechanisms to gain access to stored photos or to create more room in the workspace.

Each storage mechanism provided different advantages and disadvantages for supporting each organizational activity. The following sections describe the interactions that occurred during each organizational phase and whether or not each storage mechanism supported these interactions.

\section{Coarse-grained organization of the workspace}

All six groups made extensive use of the storage mechanisms while sorting the photos. A group typically placed each theme page in a storage bin or in a separate region of the peripheral storage area. For each photo, if it related to one of the four themes, it was stored with the appropriate theme page. If not, it was discarded into a separate storage bin or in a separate region the peripheral storage area.

In general, participants used fast, casual interactions during the coarse-grained organization of the workspace. People spent little time tidying up the theme piles. Once a photo had been classified, it was typically dragged or tossed quickly into the appropriate pile. Consequently, the large target area provided by the peripheral storage area was well
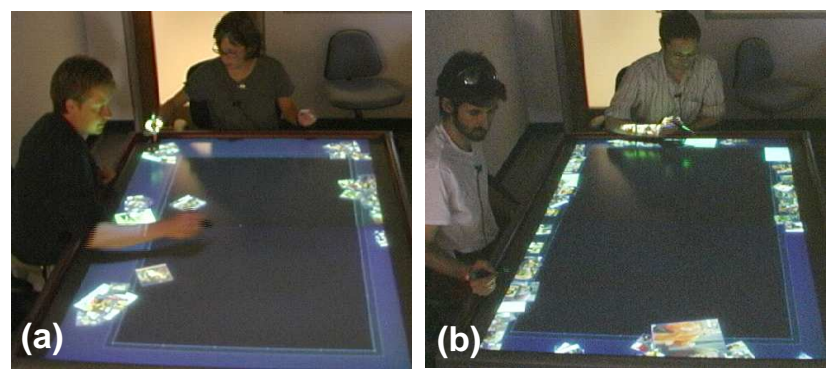

Figure 6. Teams sorting photos into theme piles in the peripheral storage area.

suited to the sorting activity. People were able to quickly drag or toss items across the long, continuous boundary and the table edge stopped the items from going beyond the storage area. When using this storage mechanism, all of the groups established four separate theme piles in the peripheral storage area and one or more piles for discarded photos that could not be classified. Two theme piles were typically established near each participant, while the piles of discarded photos were typically established along the sides where neither participant was seated (see Figure 6).

Sorting the photos using the storage bins required more accuracy because of their smaller size. Each group used a separate storage bin per theme and one or more storage bins for the discarded photos (see Figure 7). Though the sorting process could be slower because more accuracy was required, some people preferred this storage mechanism for organizing the workspace. Several participants reported that they "preferred the movable bins for organizing content and moving groups" and they felt that "having different storage spaces made grouping easier."

\section{Fine-grained organization of the workspace}

The next stage of the task involved creating the theme layouts. During this stage, photos were retrieved from the storage mechanisms and then arranged on the theme pages. When a theme layout was finished, the assembled theme layout and left-over photos were typically re-stored to create space to work on the next theme layout. This stage involved a mix of careful manipulation of the theme pages, photos, and storage mechanisms, and of casual discarding
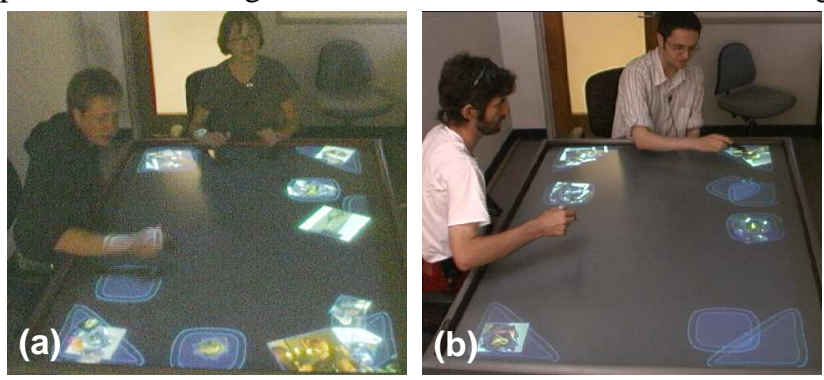

Figure 7. Teams sorting photos into theme piles in the storage bins. 

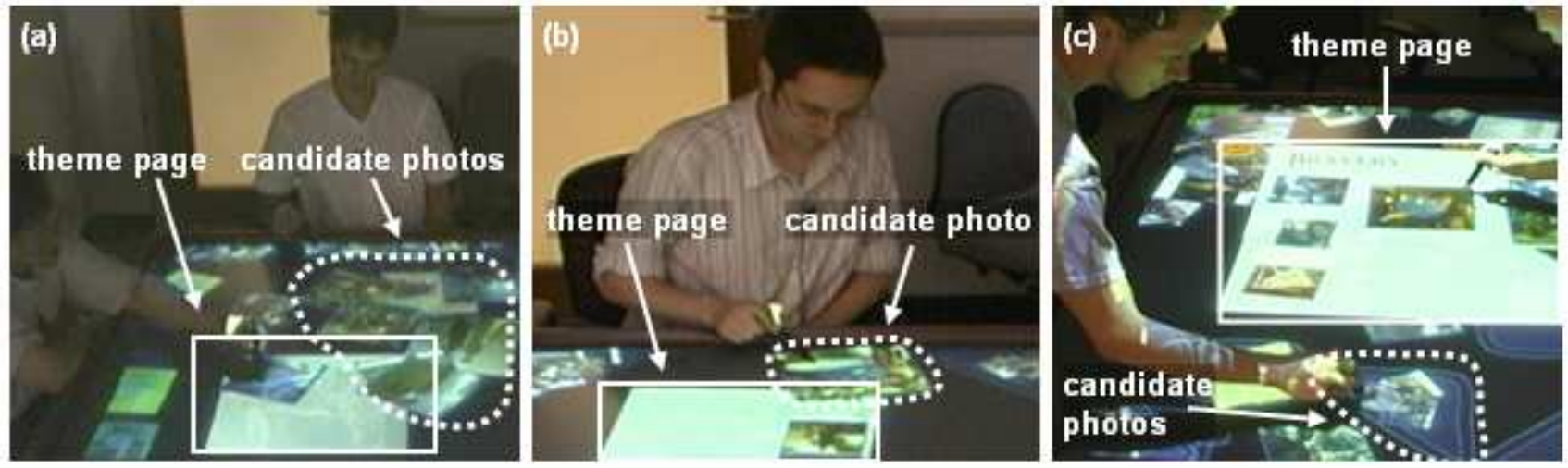

Figure 8. The three organizational methods: (a) traditional, (b) previewing, and (c) within-storage. In (a) and (b) the candidate photos are being viewed in the main workspace, but in (c) the candidate photos are inside a storage bin.

of photos that were not incorporated into the final layouts. In general, the storage bins supported this mix of activities more effectively than the peripheral storage area. The storage bins provided greater flexibility for supporting the variety of individual and collaborative working styles employed by the participants while they were creating the theme layouts.

\section{Organizing the candidate photos}

In order to create the theme layouts, participants had to choose several photos (typically 5-8) from the pile of candidate photos (typically 15-20) that the group had originally categorized into the particular theme. Participants used several methods to organize the candidate photos in the workspace to facilitate the photo selection process: traditional, previewing, and within-storage (see Figure 8). The traditional method involved participants retrieving a group of candidate photos from storage and then choosing from the pile of full-sized photos in the workspace. The previewing method involved participants retrieving one candidate photo at a time from storage for full-size viewing before deciding whether or not it was appropriate for the layout. If so, the photo was added to the layout. If not, it was re-stored and another candidate photo was retrieved until the layout was complete. The withinstorage method involved participants choosing candidate photos directly from within the storage mechanism to add to the layout.

Participants tended to move candidate photos close to the theme page, often just beside it, allowing them to make easy visual comparisons between the current layout and the candidates. When items were stored in the peripheral storage area, participants typically used the traditional method to compare candidate photos with the current layout (see Figure 8a). Previewing was also used, but much less often. Participants rarely used the within-storage method when using the peripheral storage area. While the photos in the peripheral storage area were very close, and thus easily viewed, people tended to want to view the candidate photos directly to one side or the other of the theme page. Since the photos stored in the peripheral storage area were typically located at the bottom of the theme page, this may explain the more frequent use of the traditional and previewing methods for choosing photos.

In contrast, storage bins easily supported all three methods of organizing candidate photos. People could move a storage bin out of way if they needed more space for creating a pile of full-sized photos or space to preview photos beside the layout. The storage bins could also be moved directly beside the theme page (see Figures $6 \mathrm{c}$ and 7a). Furthermore, the storage bins could be expanded to show more candidate photos at once. People often added photos directly to a photo layout from within a storage bin. Choosing photos from within storage allowed people to minimize the space occupied by candidate photos, thus, reducing overlaps and facilitating the photo selection process. In general, storage bins provided more support for customization of the workspace, allowing people to use the organizational method they preferred.

\section{Collaboration styles affect workspace organization}

The style of collaboration used by the different groups also affected which organizational method they used for choosing photos for the layout. Half of the groups used a divide-and-conquer strategy, with each team member working independently to create a theme layout. The remaining groups worked together on a single layout at a time.

Participants working together on the same layout tended to use the traditional and previewing methods for choosing photos, regardless of which storage mechanism was currently being used. These methods facilitated group discussion of the candidate photos better than the withinstorage method because full-sized photos were easier to share among group members than small, stored photos. Only one pair working together on a layout selected photos 

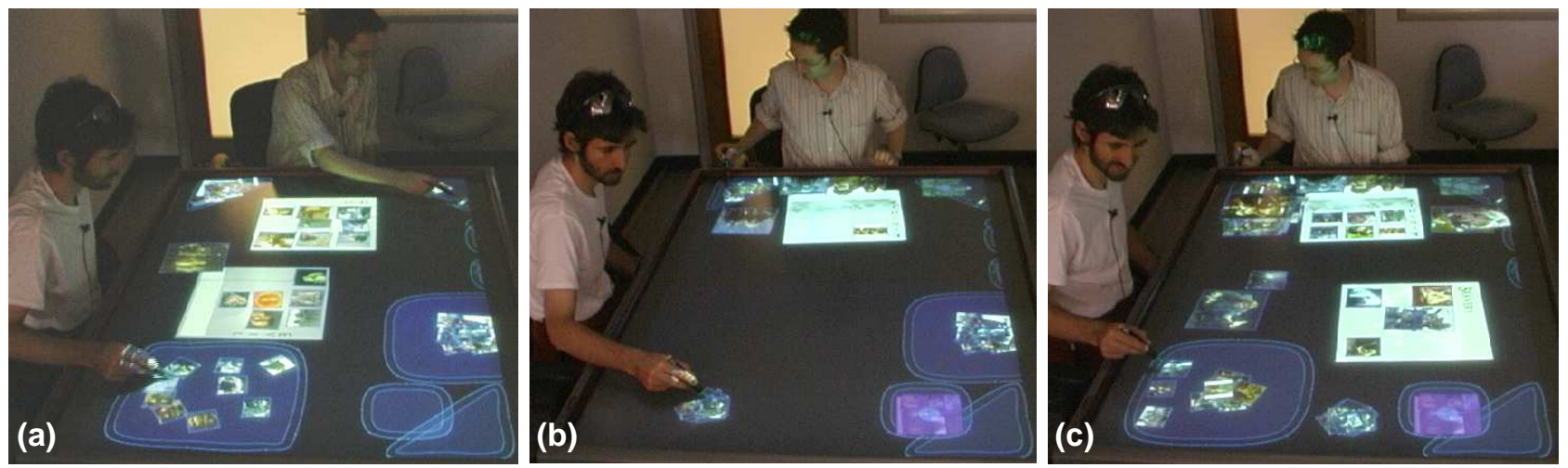

Figure 9. Localized use of the storage bins: (a) participants select photos from separate storage bins, (b) the participant on the left collapses a storage bin, and (c) then he selects photos from a different storage bin while his partner continues using the same storage bin.

directly from within storage (see Figure 8c). This pair was also the only pair that stood during their session, which may have made it easier to share the smaller items because they could easily lean over the workspace.

Participants working independently in the workspace used all three organizational methods. However, the particular method they used during each trial often depended on the current storage mechanism they were using. When using the peripheral storage area, they tended to use the traditional and previewing methods. On the other hand, when they were using the storage bins, they tended to use the within-storage method. This difference likely resulted because the peripheral storage area provided limited access to stored items across the whole workspace: as mentioned above, restricting the stored items to the periphery did not suit everyone's working style.

\section{Supporting various collaboration styles}

In general, the storage bins were more effective at supporting variations in collaboration styles than the peripheral storage area because the storage bins enabled localized storage interactions. The mobility of storage bins allowed groups working together on a layout to position a storage bin centrally so that both participants could access the stored items (see Figure 8c). Furthermore, the adjustability and mobility of storage bins allowed people to bring a pile of stored photos into and out of the main focus of activity without interfering with their partners' activities. Several participants took advantage of these features to provide easier access to stored photos and to create more space when photos were no longer needed.

An example of this behaviour is shown in Figure 9. This figure shows a sequence of interactions where a participant (on the left) first selects photos from an expanded storage bin (a), then he collapses the storage bin (b), and finally he selects photos from a second expanded storage bin (c). Notice that in (c) he has moved the first storage bin out of the way, towards the far side of the table. Also, notice that his interactions have not affected his partner's access to the stored photos in the upper left corner of the table. If the pile of photos had been in a peripheral storage area in front of him, collapsing the pile would have collapsed all photos being stored along that table edge, including the photos his partner was using. The localized behaviour of the storage bins frees each group member from worrying about interfering with their collaborators' interactions.

\section{Establishment of personal territories}

Our previous observations have shown that establishing a personal territory on the table is important for tabletop collaborators working in parallel because they tend to use this area to perform their task activities and for storing task resources they require [7]. Personal territories typically comprise the area on the table directly in front of each person. However, because the peripheral storage area occupied this area, it was unavailable for working on the layout. When using this storage mechanism, group members working in parallel typically placed their theme page just above the storage area in the main workspace and arranged their candidate photos beside the theme page.

When using the storage bins, group members working in parallel typically placed either a theme page or a storage bin that they were actively using for choosing photos directly in front of them on the table, in close proximity to the table edge. Thus, it would seem that when possible, people preferred to establish personal territories close to the table edge when working in parallel during the layout task. The fixed nature of the peripheral storage area prevented this behaviour.

In summary, both storage mechanisms supported casual piling during the collaborative layout task. It appears, though, that the peripheral storage was better suited to supporting the coarse-grained organizational activities that occurred during this task, while the storage bins was better 
suited to more fine-grained organizational activities. More specifically, the large, fixed nature of the peripheral storage area enabled quick, inaccurate piling common in the coarse-grained organizational activities. In contrast, the mobility and adjustability of the storage bins enabled localized piling interactions, supporting the variations of individual and collaborative working styles that occurred during the fine-grained organizational activities.

\section{Design Insights}

While the storage bins were used extensively throughout the layout sessions, observations indicated that there might have been more frequent use of move and resize if the touch interface had been more intuitive.

Since tabletop orientation issues make traditional menu choice methods problematic, we chose to use a radial marking-style menu to access the move, resize, and reshape functionalities for storage bins (Figure 8) and the resize functionality for the peripheral storage area. For example, moving a storage bin involved touching the control point to reveal the menu, then dragging the touch-point to the move menu icon, and then moving the touch-point to the new location. This menu design was used because it offered an orientation-independent control and because similar marking-style menus have been shown to be useful on other touch-input large displays [2].

However, the video data revealed that this choice could be improved upon. Using these menus did not seem to be intuitive for many participants. Several people repeatedly tried to move a storage bin by touching an empty area in the storage bin and dragging. This may be because the photos and theme pages could be moved by touch and drag. Several people also tried to resize the storage areas by dragging the control point itself instead of selecting the resize menu. Providing direct-touch interactions that involve dragging on any empty area inside the storage bin to move it and dragging on a control point to resize would appear to provide a more intuitive interaction based on our participants' behaviour. Few people attempted to reshape the storage bins; thus, it may have been sufficient to provide the move and resize features.

How stored items are handled during resizing operations also needs to be considered. Collapsing a storage bin currently uses a physical metaphor of collecting up the

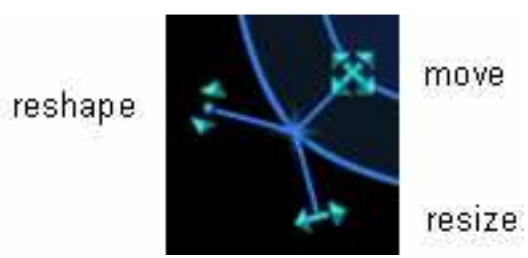

Figure 10. The marking-style menu for the storage bin.
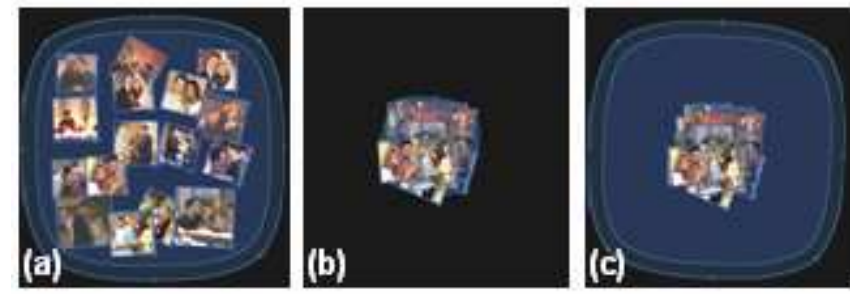

Figure 11. Collapsing and expanding storage bins: (a) the initial storage bin, (b) the collapsed storage bin, (c) the storage bin re-expanded.

stored items as the boundary sweeps inward, pushing photos toward the center. However, when the storage bin is later expanded, the photos remain clustered in the center (see Figure 11). This metaphor facilitates creating space for new items; however, it requires manually spreading out the clustered items if someone wants to later search the items.

One possible alternative to the current expand/collapse interaction is a "stretchy fabric" metaphor, that would keep stored items at the same relative distance from each other and the storage center as the boundary moves. Another alternative is to return all displaced items to their original locations when a pile is subsequently expanded after a collapse. These alternatives need further investigation.

Including both spatial and temporal transitions for scaling may be unnecessary. Participants typically placed items either completely inside or completely outside of the storage bins, rarely leaving them in the transition zone. If a photo was tossed into a storage bin and it stopped in the transition zone, people typically pushed it fully inside. Thus, it appears that scaling transitions with the timing mechanism only may be sufficient for boundary crossings. Timed scaling alone provides a smooth visual effect and eliminates the spatial transition zone freeing up additional space.

Creating a hybrid storage mechanism could provide the benefits of both mobile and peripheral storage mechanisms. Extending the mobile storage bin design to allow it to 'dock' to the workspace edge would provide the advantages of both storage mechanisms (see Figure 12). The concept of an attachable storage bin is similar to a toolbar that can float or be docked to a window edge, often seen in many

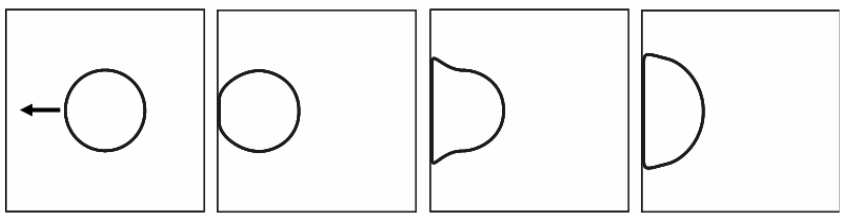

Figure 12. An attachable storage bin attaching to the edge of the workspace. 
popular development applications.

While participants found storage bins useful for selecting photos during the layout task, enhancing them with tools that provided pile management features could provide further support for organizing and searching large collections of items. Several participants commented that they would have liked to be able to automatically arrange the stored photos so they did not overlap.

Pile management techniques could help users automatically spread the pile for easier visual scanning. For example, stored items could be arranged in a grid formation, potentially ordered by some criteria, such as the date of a photo [1] or by how recently an item was stored. Alternatively, an automatic spacing algorithm could be used to spread the pile based on the size of the storage bin.

\section{Conclusions}

We have introduced storage bins, an interface component that combines storage container capabilities with mobility and flexibility. The design goals for storage bins arose from observations of how people make use of casual piles during collaboration on tables with traditional paper based media. Casual piles with traditional media help organize subtasks, maintain a grouped structure while supporting individual item selection, and are used in many locations for different purposes. Based on these properties, we designed storage bins to:

- allow the addition and removal of items individually or in groups without requiring the extra operations associated with grouping and ungrouping,

- augment traditional media capabilities by scaling stored items, thus improving space usage,

- are adjustable both in size and shape, and

- have a subdued visual appearance that clearly indicates their presence but without attracting undue attention.

Since previous casual storage facilities have typically been fixed in a peripheral location of the workspace, we investigated the use of both mobile and peripheral storage. We found that people frequently took advantage of the ability to move stored items in the workspace, and that the mobile storage bins better facilitated different individual and collaborative working styles. On the other hand, we did notice that for specific tasks, such as sorting, the peripheral storage was very effective.

In general, storage bins appear to provide tabletop collaborators more flexible support for performing task activities associated with fine-grained organization of the workspace, while peripheral storage mechanisms provide better support for more the casual interactions involved in coarse-grained organization of the workspace. As a result we recommend incorporating into mobile storage bins a docking ability to enable people to gain the benefits of peripheral storage when desired.

\section{Acknowledgements}

We gratefully thank our funding providers: Natural Sciences and Engineering Research Council (NSERC), Alberta's Informatics Circle of Research Excellence (iCORE), Alberta Ingenuity, and the Canadian Foundation for Innovation (CFI). We would also like to thank researchers from the Interactions Lab at the University of Calgary for their insightful comments on this work.

\section{References}

1. Bauer, D., Fastrez, F., \& Hollan, J. (2004). Computationally- Enriched 'Piles' for Managing Digital Photo Collections. In Proceedings of Visual Languages and Human-Centric Computing 2004.

2. Guimbretière, F., Stone, M., \& Winograd, T. (2001). Fluid Interaction with High-resolution Wall-size Displays. In Proceedings of ACM Symposium on User Interface Software and Technology (UIST)'01, pp. 2130.

3. Kruger, R., Carpendale, M.S.T., Scott, S.D., Greenberg, S. (in press). Roles of Orientation in Tabletop Collaboration: Comprehension, Coordination and Communication. In Journal of Computer Supported Collaborative Work. (accepted July 2004).

4. Malone, T.W. (1983). How do people organize their desks?: Implications for the design of office information systems. ACM Transactions on Information Systems (TOIS), 1, pp. 99-112.

5. Rekimoto, J. and Saitoh, M. (1999). Augmented Surfaces: A Spatially Continuous Work Space for Hybrid Computing Environments. In Proceedings of the ACM Conference on Human Factors in Computing Systems (CHI) '99, pp. 378-385.

6. Robertson, G., Horvitz, E., Czerwinski, M., Baudisch, P., Hutchings, D., Meyers, B., Robbins, D., and Smith, G. (2004). Scalable Fabric: Flexible Task Management. In Proceedings of Advanced Visual Interfaces (AVI)'04, pp. 85-89.

7. Scott, S.D., Carpendale, M.S.T, \& Inkpen, K.M. (2004). Territoriality in Collaborative Tabletop Workspaces. In Proceedings of the ACM Conference on ComputerSupported Cooperative Work (CSCW)'04, November 610, 2004, Chicago, IL, USA, pp. 294-303. 
8. Shen, C., Lesh, N., Vernier, F., Forlines, C., \& Frost, J. (2002), Sharing and Building Digital Group Histories. In Proceedings of the ACM Conference on ComputerSupported Cooperative Work (CSCW) 2002, pp. 324333.

9. Streitz, N.A., Geißler, J., Holmer, T., Konomi, S., Müller-Tomfelde, C., Reischl, W., Rexroth, P., Seitz, P., Steinmetz, R., Steinmetz, R., and Steinmetz, R. (1999). i-LAND: An interactive Landscape for Creativitiy and Innovation. In Proceedings of the the ACM Conference on Human Factors in Computing Systems (CHI) '99, pp. 120-127.
10.Tse, E. and Greenberg, S. (2004) Rapidly Prototyping Single Display Groupware through the SDGToolkit. In Proceedings of the $5^{\text {th }}$ Australasian User Interface Conference, Volume 28 in the CRPIT Conferences in Research and Practice in Information Technology Series, (Dunedin, NZ January), Australian Computer Society Inc., p101-110.

11.Wellner, P. (1993). Interacting with Paper on the DigitalDesk. Communications of the ACM, 36(7), pp. 86-96. 